Article

\title{
Risk Reserve Constrained Economic Dispatch Model with Wind Power Penetration
}

\author{
Wei Zhou *, Hui Sun and Yu Peng \\ Department of Electrical and Electronics Engineering, Dalian University of Technology, Dalian, \\ 116024, China; E-Mails: dutshui@dlut.edu.cn (H.S.); plyw@sina.com (Y.P.) \\ * Author to whom correspondence should be addressed; E-Mail: zhouwei-ee@163.com; \\ Tel.: +86-411-8470-6716; Fax: +86-411-8470-6716.
}

Received: 30 October 2010; in revised form: 26 November 2010 / Accepted: 1 December 2010 / Published: 7 December 2010

\begin{abstract}
This paper develops a modified economic dispatch (ED) optimization model with wind power penetration. Due to the uncertain nature of wind speed, both overestimation and underestimation of the available wind power are compensated using the up and down spinning reserves. In order to determine both of these two reserve demands, the risk-based up and down spinning reserve constraints are presented considering not only the uncertainty of available wind power, but also the load forecast error and generator outage rates. The predictor-corrector primal-dual interior point (IP) method is utilized to solve the proposed ED model. Simulation results of a system with ten conventional generators and one wind farm demonstrate the effectiveness of the proposed method.
\end{abstract}

Keywords: wind power; risk; economic dispatch; spinning reserve; interior point method

\section{Introduction}

With increasing fuel prices and environmental pollution, it has become apparent that wind energy should be considered a good alternative to thermal energy power generation. However, unlike conventional generation, the wind power output depends on the wind speed, which is related to climatological and micrometeorological parameters. Due to the limited predictability and intermittency of wind speed, wind power is commonly regarded as problematic for power system operation. It will significantly influence the normal operation of power systems because the actual output of wind power 
usually deviates from the scheduled value [1]. If the actual output is smaller than the scheduled one, the energy shortage will increase the risk of load shedding in power systems. On the contrary, an energy surplus will increase the risk of wind energy waste. Therefore, it is necessary to allocate additional spinning reserve in the ED issue for any possible unexpected generation deficit and surplus. In [2-4], the authors used some efficient optimization algorithms to investigate the relationships between operation cost, spinning reserve requirements and wind power penetration level. The additional spinning reserve due to wind power generation was taken into consideration in the constraint and the spinning reserve requirement was treated as a simple fraction of the total wind power generation which was assumed from experimental results. The amount of spinning reserve equal to wind power capacity is absolutely safe for system operation, but it is a waste because the wind power output doesn't operate at the rated value all the time. Moreover, a redundant reserve will also increase the operational cost, so this kind of deterministic generation scheduling is conservative at high wind penetration levels. Summing up the above, investigating the relationship between risk, cost, wind power penetration and reserve level is an urgent issue for the sake of safe and economic operation.

The uncertainty of wind power has been considered in several ED optimization models. Some are based on the fuzzy-set theory [5-7], while others are based on the stochastic method [8-14]. In the proposed ED model of [5] and [6], a fuzzy membership function was defined to describe the relationship between system security level and wind power penetration/operation cost. The dispatcher's attitude toward the risk and cost was involved in the dispatch model of [5]. Reference [6] formulated a bi-objective ED problem and its optimization was achieved in a compromise between risk and cost. However, the spinning reserve, which is very indispensable for safe operation, is not considered in the model. Besides, Hong and Li [7] pointed out that the fuzzy-set theory could be used to model uncertainties of wind power involving climate for the short-term active power scheduling. When the ED issue is investigated with stochastic model of wind power, different probability density functions have been involved. Reference [8] developed a model with wind energy conversion system generators, in which a Weibull probability density function was used to characterize the stochastic wind speed [9]. Both risk of overestimation and cost of underestimation of available wind power have been considered by introducing reserve cost and penalty cost into the objective function. It is worth noting that reserve requirement constraints were not mentioned in the model. Since the Weibull distribution indicates the average wind speed in one year, it can be easily found that this ED model is based on the long-term schedule. However, different from long-term schedule, forecasting techniques should be utilized when considering the actual short-term conditions. In order to describe the uncertainty of wind power, a corresponding stochastic model of wind speed/wind power forecast error should be formulated. Reference [10] considered an optimal power flow wind-thermal coordination model similar to that reported in [8], but different approaches were utilized to estimate the probability density function of wind power forecast error. In [11], the wind power is subject to a normal distribution with available forecasted wind power as its expected value, and then the Monte Carlo simulation based on the Latin hypercube sampling technique was employed to generate possible wind power volatility scenarios. Bouffard and Galiana [12] proposed a stochastic security planning methods which can better cope with the nature of wind generation while maintaining or even improving the current reliability and economic performance of power systems. Soder [13] proposed a methodology to 
analyze the operation planning with an emphasis on maintaining the reserves, which has considered wind speed forecast uncertainties, system load forecast uncertainties, ramp rates of thermal units and spinning reserves, etc. Siahkali and Vakilian [14] described the uncertainties of the wind speed by a Gaussian distribution and simulated it by a sampling approach. As a reasonable distribution of wind speed forecast error for short-term ED issue, the normal distribution function is also applied in our paper. Besides, an effective treatment method is adopted in which the required reserve is determined by satisfying a given risk threshold [15].

Based on the stochastic distribution of wind speed, a modified ED model including wind power penetration is presented. The overestimation and underestimation of the wind power generation are considered in the form of a reserve cost penalty, while satisfying the risk reserve constraints. According to the proposed model, all the available wind power will be utilized within a given risk level. The paper is structured as follows: in Section 1, an introduction is given. In Section 2, the proposed ED model is introduced. In Section 3, stochastic characterizations of wind power and load demand are described. In order to quantify the effect of random variables on system risk, the normal distributions of wind speed and load forecast errors are assumed. Section 4 points out the method for solving the proposed ED model. In Section 5, the results of a ten-generator system containing a wind farm are analyzed. Finally, conclusions are drawn and discussed.

\section{Risk Reserve Constrained Optimization Model}

Because of the uncertainty of the wind energy available at any given time, this paper considers the costs associated with overestimation and underestimation of wind power generation. The shortage and surplus of wind power can be compensated by the up and down spinning reserves. Furthermore, indispensable spinning reserves are also used due to the generator outages and load forecast error.

The objective function is expressed as follows:

$$
\min \sum_{n=1}^{N} C_{n}\left(P_{n}\right)+\sum_{n=1}^{N_{w}} C_{w, n}\left(W_{n}\right)+\sum_{n=1}^{N} C_{p, n}\left(R_{d, n}\right)+\sum_{n=1}^{N} C_{r, n}\left(R_{u, n}\right)
$$

In the above function, the first term is the total fuel cost of conventional generators. Generally, the fuel cost will be a second order polynomial function as follows:

$$
C_{n}\left(P_{n}\right)=a_{n} \times P_{n}^{2}+b_{n} \times P_{n}+C_{n}
$$

where $P_{n}$ is the power from $n$th conventional generator; $N$ is the number of conventional generators; $a_{n}, b_{n}, c_{n}$ are constants of fuel cost function of $n$th conventional generator.

The second term is the cost for wind-powered generators. In market-oriented liberalized power systems, the wind power is privately owned by a non-utility, so the incremental costs brought about by wind power generation cannot be neglected in the ED model. It is based on the special contractual agreements and can be given by:

$$
C_{w, n}\left(W_{n}\right)=W_{n} \times d_{n}
$$

where $W_{n}$ is the scheduled output of the $n$th wind-powered generator; $N_{w}$ is the number of wind turbine generator; $d_{n}$ is the direct cost coefficient for the $n$th wind-powered generator. In order to simplify the problem, both the output and direct cost coefficient for each wind turbine generator are 
assumed to be identical in the wind farm. So the total amount of wind power generation in a wind farm is $W=n W_{n}$.

The third term indicates the cost of down spinning reserve required. It is a penalty cost for the case where not all the available wind power is used and load demand forecast is an overestimate of the active value. The expression is as follows:

$$
C_{p, n}\left(R_{d, n}\right)=R_{d, n} \times k_{p}
$$

where $R_{d, n}$ is the required down spinning reserve of the $n$th generator; $k_{p}$ is the down reserve cost coefficient.

The last term in the objective function is the cost of the up spinning reserve required, which denotes a risk cost caused by the overestimate of available wind power, the underestimate of load demand and the generator outages:

$$
C_{r, n}\left(R_{u, n}\right)=R_{u, n} \times k_{r}
$$

where $R_{u, n}$ is the required up spinning reserve of the $n$th generator; $k_{r}$ is the up reserve cost coefficient.

Compared to the model in [8], there are two modifications in this paper: (1) the required up and down reserves in the objective function are introduced to substitute the expected surplus wind power and the expected deficit wind power; (2) the required up and down reserves are determined by the risk reserve constraints which will be explain in the following sections.

It should be noted that the up and down spinning reserve cost coefficient are identical for all the conventional generators. That is because the focus of the model in this paper is not on the economic dispatch of the spinning reserve, but on the relationship among the total reserve and generator outputs, so only the total required up spinning reserve $R_{u}$ and down spinning reserve $R_{d}$ will be calculated in this model.

Before listing all the constraints, it must be noted that this paper doesn't consider transmission losses and transmission constraints in the formulation for the sake of simplicity and to avoid losing the key message of risk constraints. However, we are aware of the importance of including them and have started to incorporate them in the next phase of this research.

The system constraints are shown as follows:

(1) Power balance constraint:

$$
\sum_{n=1}^{N} P_{n}+W-P_{L}=0
$$

(2) Power operating limit of $n$th conventional generator:

$$
P_{n}^{\min } \leq P_{n} \leq P_{n}^{\max }
$$

(3) Wind power output constraint:

$$
0 \leq W \leq P_{r} N_{w}
$$

(4) Risk based up and down spinning reserve constraint:

For the sake of safe and reliable operation, system risk should be limited within an acceptable level. There are two kinds of risk constraints modeled in our paper, including the risk constraint of load 
shedding based on up spinning reserve and the risk constraint of wind energy waste based on down spinning reserve. The former can be expressed as follows:

$$
\operatorname{risk}_{u}\left(R_{u}, W, P_{n}\right) \leq \alpha
$$

If the total up spinning reserve supplied by all the conventional generators can be calculated by

$$
S R_{s u}=\sum_{n=1}^{N}\left(P_{n}^{\max }-P_{n}\right)
$$

then the total required up spinning reserve $R_{u}$ must be not more than $S R_{s u}$ :

$$
0 \leq R_{u} \leq S R_{\text {su }}
$$

For the $\mathrm{N}-1$ condition, the total up spinning reserve supplied by conventional generators can be calculated as:

$$
S R_{s u}^{m}=\sum_{\substack{n=1 \\ n \neq m}}^{N}\left(P_{n}^{\max }-P_{n}\right) \quad m=1,2 \ldots N
$$

then the total required up spinning reserve must also be not more than $S R_{s u}^{m}$ :

$$
0 \leq R_{u} \leq S R_{s u}^{m}
$$

Similar to inequality (9), the risk of wind energy waste based on down spinning reserve should be limited within a risk threshold:

$$
\operatorname{risk}_{d}\left(R_{d}, W\right) \leq \beta
$$

If the following formula indicates the total down spinning reserve supplied by all the conventional generators:

$$
S R_{s d}=\sum_{n=1}^{N}\left(P_{n}-P_{n}^{\min }\right)
$$

then the total required down spinning reserve $R_{d}$ must be not more than $S R_{s d}$ :

$$
0 \leq R_{d} \leq S R_{\text {sd }}
$$

For the $\mathrm{N}-1$ condition, the total down spinning reserve supplied by conventional generators can be calculated as:

$$
S R_{s d}^{m}=\sum_{\substack{n=1 \\ n \neq m}}^{N}\left(P_{n}-P_{n}^{\min }\right)
$$

then the total required down spinning reserve must also be not more than $S R_{s d}^{m}$ :

$$
0 \leq R_{d} \leq S R_{s d}^{m}
$$

where $P_{L}$ is the forecast value of load demand; $P_{n}^{\max }$ and $P_{n}^{\min }$ are the maximum and minimum power from $n$th conventional generator, respectively; $P_{r} N_{w}$ is the wind power capacity; $\alpha$ and $\beta$ are the risk thresholds; $S R_{s u}$ is the total up spinning reserve supplied by all the conventional generators; $S R_{s u}^{m}$ is the total up spinning reserve supplied by all the conventional generators except $m$ th conventional generator; $S R_{s d}$ is the total down spinning reserve supplied by all the conventional generators; $S R_{s d}^{m}$ is 
the total down spinning reserve supplied by all the conventional generators except $m$ th conventional generator.

This model refers to two risks in formulae (9) and (14). Both of them consider the random distributions of available wind power generation, load demand forecast error and the probability of generation forced outage. The former describes the level of system reliability using the probability of shedding load. That means the probability of the total amount of required up spinning reserve being smaller than the power generation shortfall. It is expressed in terms of total probability formula:

$$
\begin{aligned}
\operatorname{risk}_{u}\left(R_{u}, W, P_{n}\right) & =\prod_{n=1}^{N}\left(1-p r_{n}\right) \cdot \mathrm{P}\left\{R_{u} \leq \Delta P_{L}+\left(W-W_{a v}\right)\right\}+\sum_{n=1}^{N} p r_{n} \cdot \prod_{\substack{m=1 \\
m \neq n}}^{N}\left(1-p r_{m}\right) \cdot \mathrm{P}\left\{R_{u} \leq P_{n}+\Delta P_{L}+\left(W-W_{a v}\right)\right\} \\
& =\prod_{n=1}^{N}\left(1-p r_{n}\right) \cdot \mathrm{P}\left\{W_{a v}-\Delta P_{L} \leq W-R_{u}\right\}+\sum_{n=1}^{N} p r_{n} \cdot \prod_{\substack{m=1 \\
m \neq n}}^{N}\left(1-p r_{m}\right) \cdot \mathrm{P}\left\{W_{a v}-\Delta P_{L} \leq P_{n}+W-R_{u}\right\}
\end{aligned}
$$

where $p r_{n}$ is the probability of $n$th conventional generator trip; $\Delta P_{L}$ is the load forecast error, it is a random variable.

In the above formula, it is assumed that wind-powered generator does not provide a spinning reserve and the generation forced outage of wind-powered generator is not considered. The first term stands for the case of having no conventional generator trip, and the second term indicates the case of having only one conventional generator trip. The probability of two or more conventional generators trip is ignored in this model.

Similarly, the latter risk form denotes the probability of the required down spinning reserve being smaller than surplus power generation. Using the down spinning reserve constraint based on system risk, the waste of available wind power will be avoided within a given risk threshold:

$$
\operatorname{risk}_{d}\left(R_{d}, W\right)=\mathrm{P}\left\{R_{d} \leq-\Delta P_{L}+\left(W_{a v}-W\right)\right\}=1-\mathrm{P}\left\{W_{a v}-\Delta P_{L} \leq R_{d}+W\right\}
$$

Seen from formula (19) and (20), $W_{a v}-\Delta P_{L}$ exists in both of them. The correlative probability distributions will be derived in the following section.

\section{Stochastic Characterizations of Wind Power and Load Demand}

\subsection{Probability Functions of Wind Speed and Wind Power}

Wind speed and wind power prediction techniques are currently hot topics, which have attracted the attention by many researchers. In order to describe the uncertainty of wind forecasting, wind speed forecast error is represented by a density distribution function. The probability density function of the deviations between predicted and actual wind speed can be illustrated as a Gaussian distribution, which was also addressed in some previous investigations [13,16-18]. In this paper, the forecast error of wind speed is assumed to be normally distributed with a mean of zero and a standard deviation of $\sigma_{v}$, and its probability density function is given by:

$$
f_{v}(\Delta v)=\frac{1}{\sqrt{2 \pi} \sigma_{v}} \mathrm{e}^{-\frac{\Delta v^{2}}{2 \sigma_{v}^{2}}}
$$

where $v$ is the wind speed; $\Delta v$ is the forecast error of wind speed. 
For a variable speed wind turbine, the wind power generation can be obtained by the nonlinear wind speed-to-power output relationships as follows [19]:

$$
P_{w}=\left\{\begin{array}{l}
0 \quad 0 \leq v<v_{i} \text { or } v>v_{o} \\
a+b v^{3} \quad v_{i} \leq v<v_{r} \\
P_{r} \quad v_{r} \leq v \leq v_{o}
\end{array}\right.
$$

where $v_{i}$ is the cut-in wind speed; $v_{r}$ is the rated wind speed; $v_{0}$ is the cut-out wind speed; $P_{r}$ is the rated wind power from a wind-powered generator; $a=\frac{P_{r} v_{i}^{3}}{v_{i}^{3}-v_{r}^{3}}, b=\frac{P_{r}}{v_{r}^{3}-v_{i}^{3}}$.

Then the available wind power output in a wind farm is equal to:

$$
w_{a v}=P_{w} \times N_{w}
$$

where $N_{w}$ is the number of wind turbine generator.

According to formulae (22) and (23), the distribution of wind speed random variable is transformed to corresponding wind power distribution. $V$ and $W_{a v}$ are used to indicate the random variables of wind speed and available wind power output respectively. It is easy to verify that $W_{a v}$ is a mixed random variable. For the discrete portions, the probability at minimum and maximum are given in the following two formulas.

The probability of $W_{a v}=0$ can be calculated by the probability of $V<v_{i}$ or $V>v_{o}$ as follows:

$$
p_{0}=\mathrm{P}\left\{W_{a v}=0\right\}=F_{V}\left(v_{i}\right)+\left(1-F_{V}\left(v_{o}\right)\right)=\Phi\left(\frac{v_{i}-\bar{v}}{\sigma_{v}}\right)+1-\Phi\left(\frac{v_{o}-\bar{v}}{\sigma_{v}}\right)
$$

while the probability of $W_{a v}=\mathrm{P}_{r} \cdot N_{w}$ can be obtained by the probability of $v_{r} \leq V \leq v_{o}$ :

$$
p_{r}=\mathrm{P}\left\{W_{a v}=\mathrm{P}_{r} \cdot N_{w}\right\}=F\left(v_{o}\right)-F\left(v_{r}\right)=\Phi\left(\frac{v_{o}-\bar{v}}{\sigma_{v}}\right)-\Phi\left(\frac{v_{r}-\bar{v}}{\sigma_{v}}\right)
$$

The random variable $W_{a v}$ is continuous between minimum and maximum. Since the wind speed has a given distribution, it is necessary to convert that distribution to a wind power distribution. The transformation can be accomplished according to formulas (22) and (23) as follows:

$$
W_{a v}=g(V)=\left(a+b V^{3}\right) \cdot N_{w}
$$

Because both the monotonic and derivative function $g(V)$ and the probability density function of random variable $V$ are given, the probability density function of $W_{a v}=g(V)$ can be obtained according to the theory of Probability and Statistics. The calculation expression is as follows:

$$
f_{W_{a v}}\left(w_{a v}\right)=f_{v}\left(g^{-1}\left(w_{a v}\right)\right) \cdot\left|\frac{d g^{-1}\left(w_{a v}\right)}{d w_{a v}}\right|=\frac{1}{3 \sqrt{2 \pi} \sigma_{v} b^{\frac{1}{3}} N_{w}^{\frac{1}{3}}}\left(w_{a v}-a N_{w}\right)^{-\frac{2}{3}} \cdot e^{-\left(\frac{\left.\left(\frac{w_{a v}-a N_{w}}{b N_{w}}\right)^{\frac{1}{3}}-\bar{v}\right)^{2}}{2 \sigma_{v}^{2}}\right.}
$$

\subsection{Probability Function of Load Forecast Error}

Actual load or load forecasting error can be assumed to be a random variable. Normal distribution has been frequently used and employed to model actual load in a number of research works [20,21]. In 
this paper the load forecast error is considered as a normally distributed random variable, and its probability density function is:

$$
f_{\Delta P_{L}}\left(\Delta p_{L}\right)=\frac{1}{\sqrt{2 \pi} \sigma_{L}} e^{-\frac{\Delta p_{L}^{2}}{2 \sigma_{L}^{2}}}
$$

where $\Delta p_{L}$ is the load forecast error. It is a realization of $\Delta P_{L}$.

\subsection{Total Stochastic Characterization}

In this section, a new random variable $Z$ is defined to calculate the probability density function of the difference between $W_{a v}$ and $\Delta P_{L}$ :

$$
Z=W_{a v}-\Delta P_{L}
$$

where $\Delta P_{L}$ is a given normally distributed random variable, the probability density function of $W_{a v}$ can be derived by a transformation from wind speed to wind power generation, and the correlation between $W_{a v}$ and $\Delta P_{L}$ is neglected in the following calculation.

According to the convolution formula, the probability density function of random variable $Z$ can be obtained using the distributions of random variable $W_{a v}$ and $\Delta P_{L}$ :

$$
\begin{aligned}
f_{Z}(z)= & p_{0} \cdot f_{\Delta P_{L}}(z)+p_{r} \cdot f_{\Delta P_{L}}\left(z-P_{r} N_{w}\right)+\int_{0}^{P_{r} N_{w}} f_{W_{a v}}\left(w_{a v}\right) \cdot f_{\Delta P_{L}}\left(z-w_{a v}\right) d w_{a v} \\
= & \left(\Phi\left(\frac{v_{i}-\bar{v}}{\sigma_{v}}\right)+1-\Phi\left(\frac{v_{o}-\bar{v}}{\sigma_{v}}\right)\right) \cdot \frac{1}{\sqrt{2 \pi} \sigma_{L}} e^{-\frac{z^{2}}{2 \sigma_{L}^{2}}}+\left(\Phi\left(\frac{v_{o}-\bar{v}}{\sigma_{v}}\right)-\Phi\left(\frac{v_{r}-\bar{v}}{\sigma_{v}}\right)\right) \cdot \frac{1}{\sqrt{2 \pi} \sigma_{L}} e^{-\frac{\left(z-P_{r} N_{w}\right)^{2}}{2 \sigma_{L}^{2}}} \\
& +\int_{0}^{P_{r} N_{w}} \frac{\left(w_{a v}-a \cdot N_{w}\right)^{-\frac{2}{3}}}{6 \pi \sigma_{v} \sigma_{L} b^{\frac{1}{3}} N_{w}^{\frac{1}{3}}} \cdot \mathrm{e}^{-\left(\frac{\left.\left(\frac{w_{v}-a \cdot N_{w}}{b N_{w}}\right)^{\frac{1}{3}}-\bar{v}\right)^{2}}{2 \sigma_{v}^{2}}-\frac{\left(z-w_{a v}\right)^{2}}{2 \sigma_{L}^{2}}\right.} d w_{a v}
\end{aligned}
$$

then the corresponding cumulative distribution function can also be obtained by calculating the integral of function $f_{z}(z)$ :

$$
\begin{aligned}
F_{Z}(z) & =\left(\Phi\left(\frac{v_{i}-\bar{v}}{\sigma_{v}}\right)+1-\Phi\left(\frac{v_{o}-\bar{v}}{\sigma_{v}}\right)\right) \cdot \Phi\left(\frac{z}{\sigma_{L}}\right)+\left(\Phi\left(\frac{v_{o}-\bar{v}}{\sigma_{v}}\right)-\Phi\left(\frac{v_{r}-\bar{v}}{\sigma_{v}}\right)\right) \cdot \Phi\left(\frac{z-P_{r} N_{w}}{\sigma_{L}}\right) \\
& +\int_{-\infty}^{z}\left[\int_{0}^{P_{r} N_{w}} \frac{\left(w_{a v}-a \cdot N_{w}\right)^{-\frac{2}{3}}}{6 \pi \sigma_{v} \sigma_{L} b^{\frac{1}{3}} N_{w}^{\frac{1}{3}}} \cdot \mathrm{e}^{-\frac{\left(\left(\frac{w_{v}-a \cdot N_{v}}{b N_{w}}\right)^{\frac{1}{3}}-\bar{v}\right)^{2}}{2 \sigma_{v}^{2}}-\frac{\left(t-w_{v v}\right)^{2}}{2 \sigma_{L}^{2}}} d w_{a v}\right] d t
\end{aligned}
$$

Both of the above formulas will be solved by numerical integration methods because of the complexity.

\section{Method for Solving the Proposed ED Model}

As this paper focuses only on the optimal model of ED issue but not on the optimization method, a classical optimization technique can be adopted. For this typical nonlinear programming problem, 
the IP method has proven to be a computationally viable alternative. This paper uses the predictor-corrector primal-dual IP algorithm which is explained in [22,23]. 


\section{Numerical Simulations}

To verify the feasibility and rationality of the proposed ED method, a test system with ten conventional generators and one wind farm is studied. The forecast value of load demand is set to 2,250 MW, and the standard deviation of load forecast error is assumed to be $45 \mathrm{MW}$. There are 100 wind turbine generators in the wind farm, and the rated power of each wind turbine generator is 2 MW. Critical wind speed parameters of $v_{i}=4 \mathrm{~m} / \mathrm{s}, v_{r}=12.5 \mathrm{~m} / \mathrm{s}$ and $v_{o}=20 \mathrm{~m} / \mathrm{s}$ are used. It is assumed that the forecast value of wind speed is $8.5 \mathrm{~m} / \mathrm{s}$, which indicates the expectation of normally distributed wind speed. The corresponding standard deviation is set to $10 \%$ of mean value. During the calculation of minimum generation cost, the risk threshold $\alpha$ is set to 0.03 . The risk threshold $\beta$ is set to be equal to $\alpha$. The direct cost of wind energy is $50 \mathrm{USD} / \mathrm{MWh}$. The up and down spinning reserve cost coefficients are 2.4 USD/MWh and $30 \mathrm{USD} / \mathrm{MWh}$, respectively. The generator constants of fuel cost function and the active power limits are shown in [24]. From this reference, it can be seen that the $10^{\text {th }}$ conventional generator cannot be optimized because its upper and lower powers are both equal to $55 \mathrm{MW}$. So there are nine conventional generators left which will be scheduled in the optimization problem. The probability of conventional generator trip is listed in Table 1.

Table 1. Probability of conventional generator trip.

\begin{tabular}{cccc}
\hline G & $\boldsymbol{P r}$ & G & $\boldsymbol{P r}$ \\
\hline 1 & 0.0008 & 6 & 0.0005 \\
2 & 0.002 & 7 & 0.0008 \\
3 & 0.0003333 & 8 & 0.002 \\
4 & 0.002 & 9 & 0.00033 \\
5 & 0.002 & 10 & 0.002 \\
\hline
\end{tabular}

Table 2. Optimal solution using predictor-corrector IP method.

\begin{tabular}{cccc}
\hline $\mathbf{G}$ & $\mathbf{P}(\mathbf{M W})$ & $\mathbf{G}$ & $\mathbf{P}(\mathbf{M W})$ \\
\hline 1 & 470.0 & 7 & 130.0 \\
2 & 460.0 & 8 & 50.51 \\
3 & 340.0 & 9 & 20.00 \\
4 & 230.5 & $\mathrm{~W}$ & 91.01 \\
5 & 243.0 & $R_{u}$ & 129.5 \\
6 & 160.0 & $R_{d}$ & 62.52 \\
\hline
\end{tabular}

Table 3. Computational performances of two IP algorithms.

\begin{tabular}{cccc}
\hline Method & Cost(\$) & Iter & Time(s) \\
\hline IP & 59433 & 21 & 20.71 \\
PCIP & 59432 & 13 & 14.68 \\
\hline
\end{tabular}

\subsection{Computational Performance of the Predictor-Corrector IP Method}

Table 2 shows the optimal solution under the foregoing condition. It includes the scheduled power output of conventional generators, the output of wind farm and required up/down spinning reserve. 
The complementarity gap variation process of predictor-corrector IP algorithm is indicated in Figure 1. Table 3 demonstrates the computational performance comparison of pure IP algorithm and predictorcorrector IP algorithm. From the results, it can be concluded that the predictor-corrector IP algorithm which is adopted in this paper is more efficient than the pure IP algorithm while guaranteeing identical accuracy.

Figure 1. The iteration curve of complementarity gap.

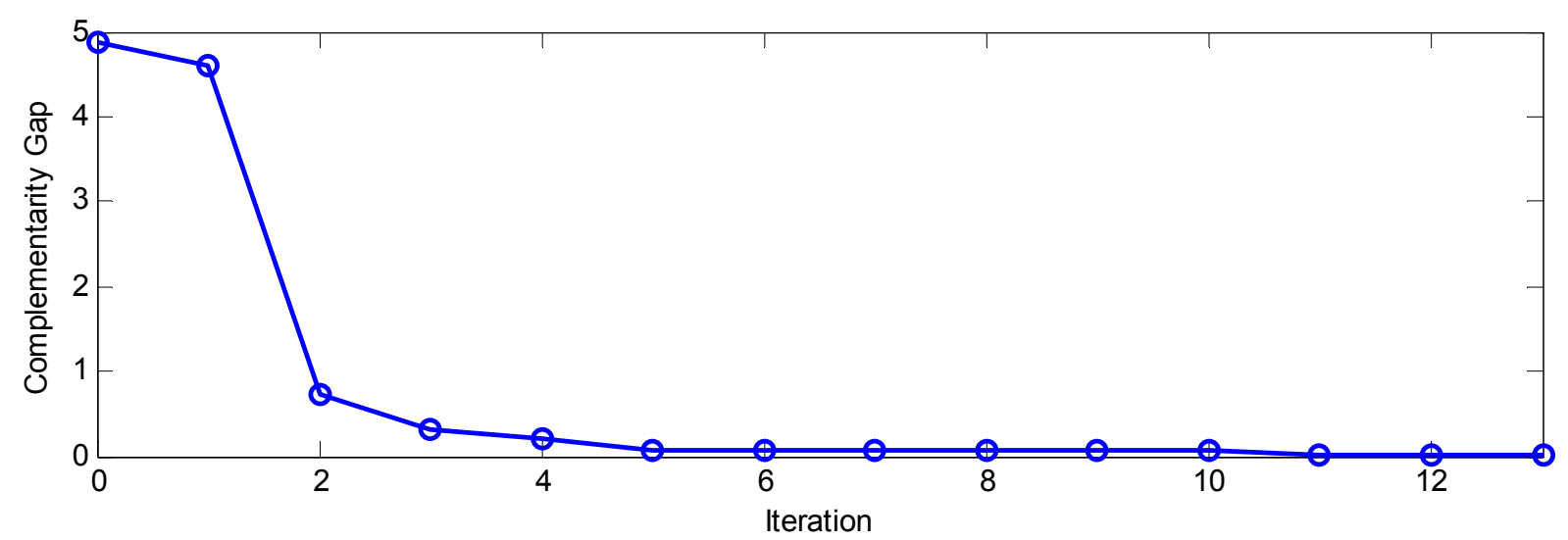

Figure 2. The total generation cost as a function of $\alpha$.

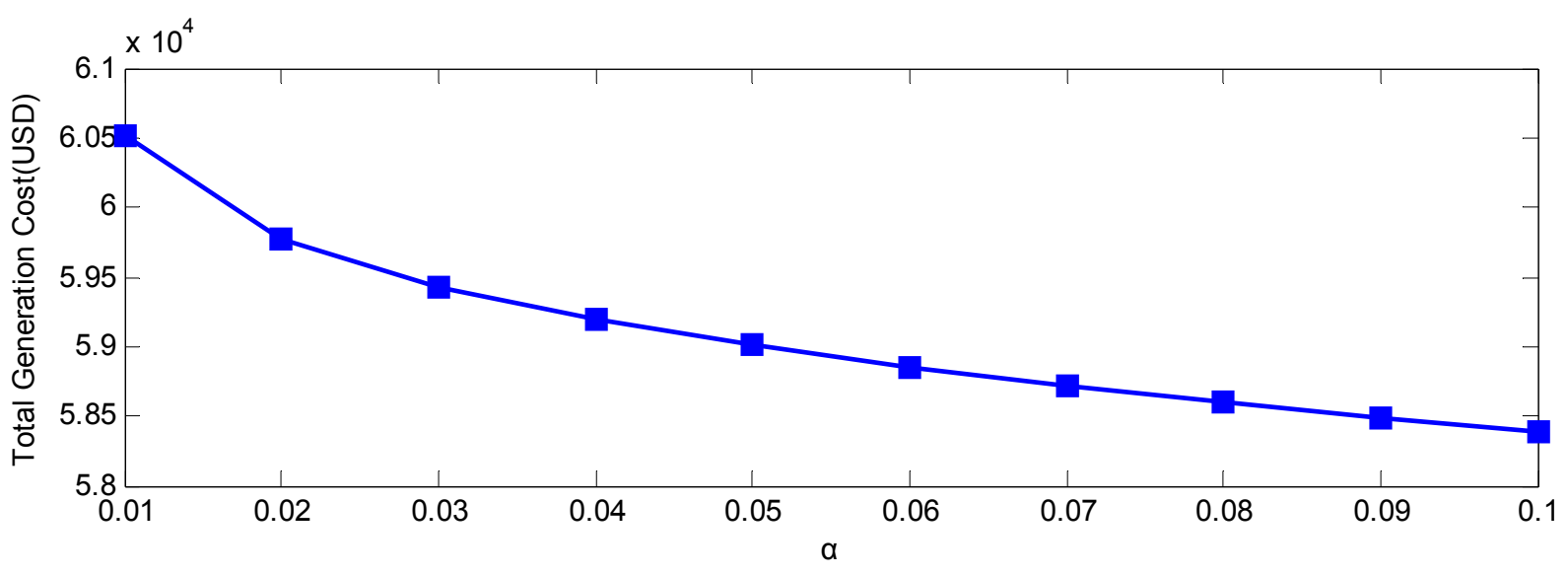

Figure 3. The scheduled wind power output as a function of $\alpha$.

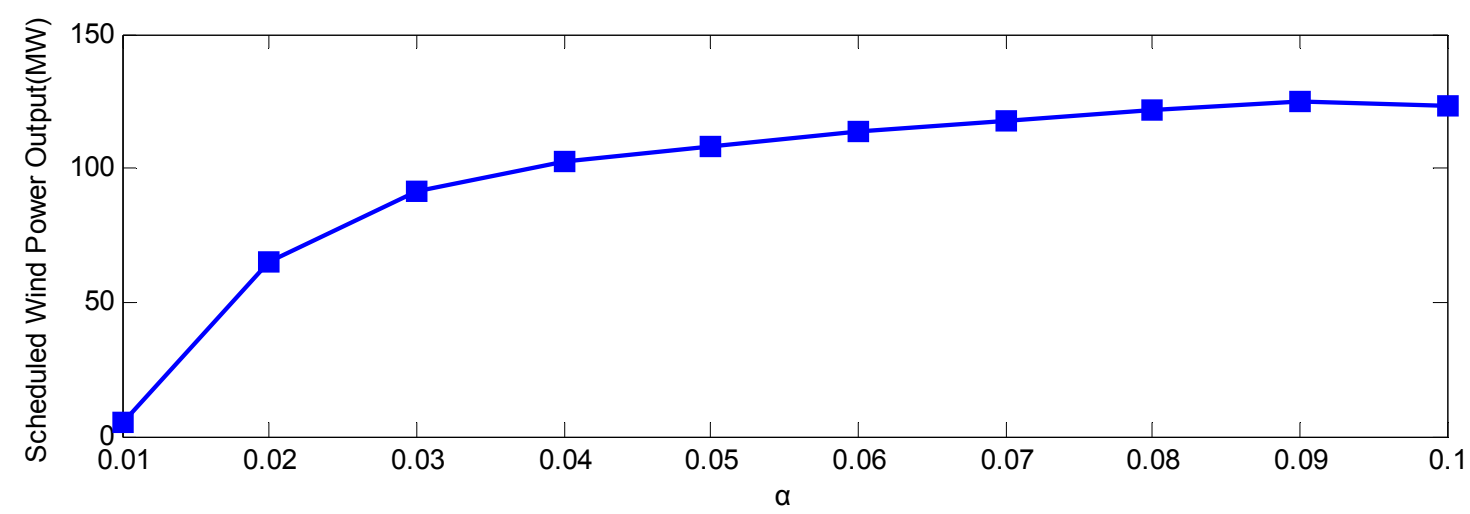


Figure 4. The required spinning reserve as a function of $\alpha$.

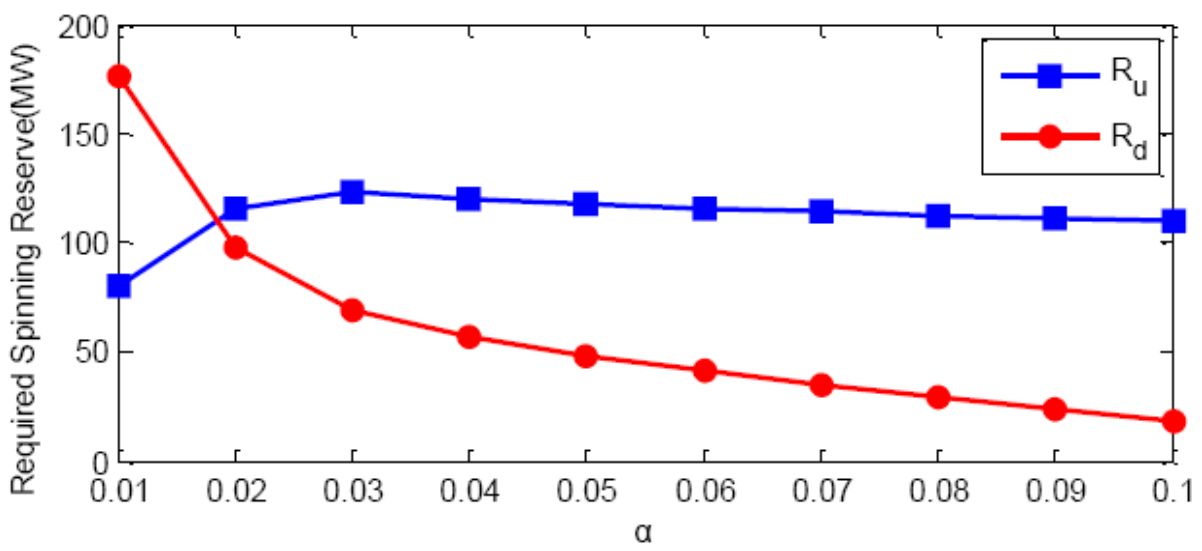

\subsection{Effects of Risk Threshold on the Optimal Outputs}

To begin some investigation of the proposed model, it is necessary to illustrate the influence of various risk threshold $\alpha$ on the solutions of ED problem. As shown in Figure 2, the objective function decreases when $\alpha$ increases. That means the reduction of system security will bring some economic benefits. From Figure 3, it can be seen that the scheduled wind power output increases quickly when $\alpha$ varies from 0.01 to 0.03 , after that it increases slowly. Figure 4 shows that the required up spinning reserve also increases as $\alpha$ rises till a value of 0.03 , but then the up spinning reserve needed by power system can't further increase. To sum up, when $\alpha$ is small, there is no enough up spinning reserve available, so the scheduled amount of wind power must be reduced to ensure the safe operation of the power system and the required up spinning reserve for compensating the uncertain wind energy will also drop. After $\alpha$ reaches 0.03 , the scheduled output of wind-power generators will increase slowly due to the limitation of up spinning reserve supply. Differing from the variation trend of required up spinning reserve, the required down spinning reserve always drops as $\alpha$ increases. The reason is that the increased amount of scheduled wind power generation will possibly lead to more waste of wind energy when it is larger than the actual output of wind power. Even though the scheduled wind power output doesn't increase any more, the increased $\alpha$ will also cause a reduction of required down spinning reserve.

Figure 5. The total generation cost as a function of up reserve cost coefficient.

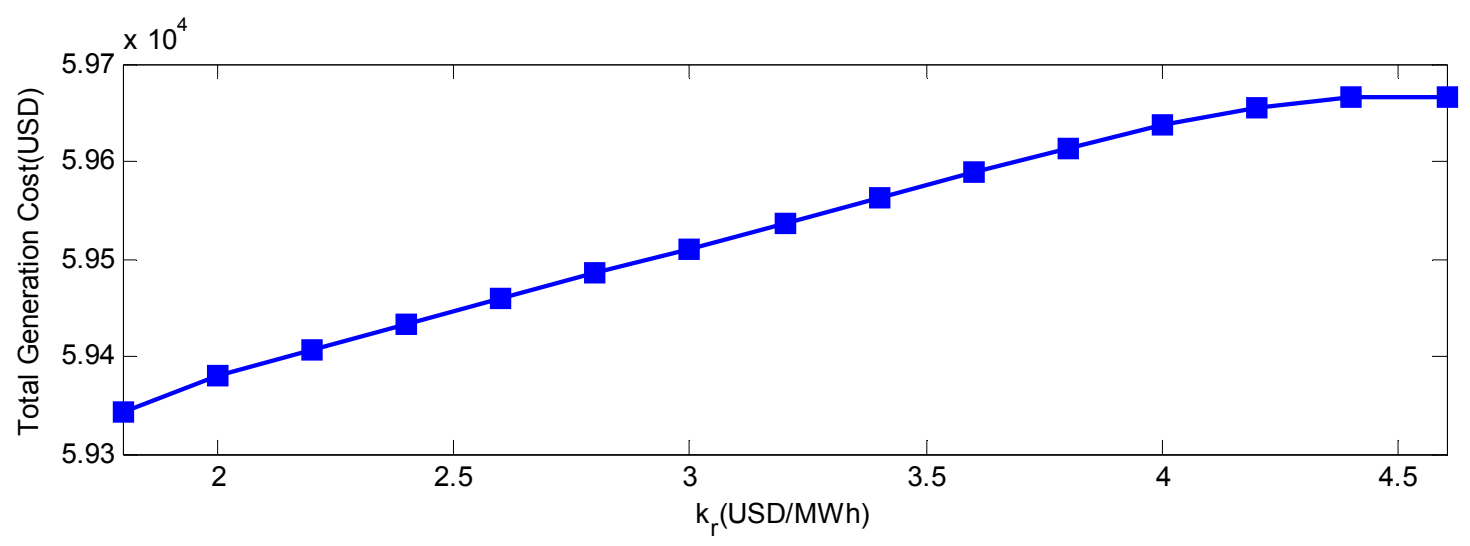


Figure 6. The scheduled wind power output as a function of up reserve cost coefficient.

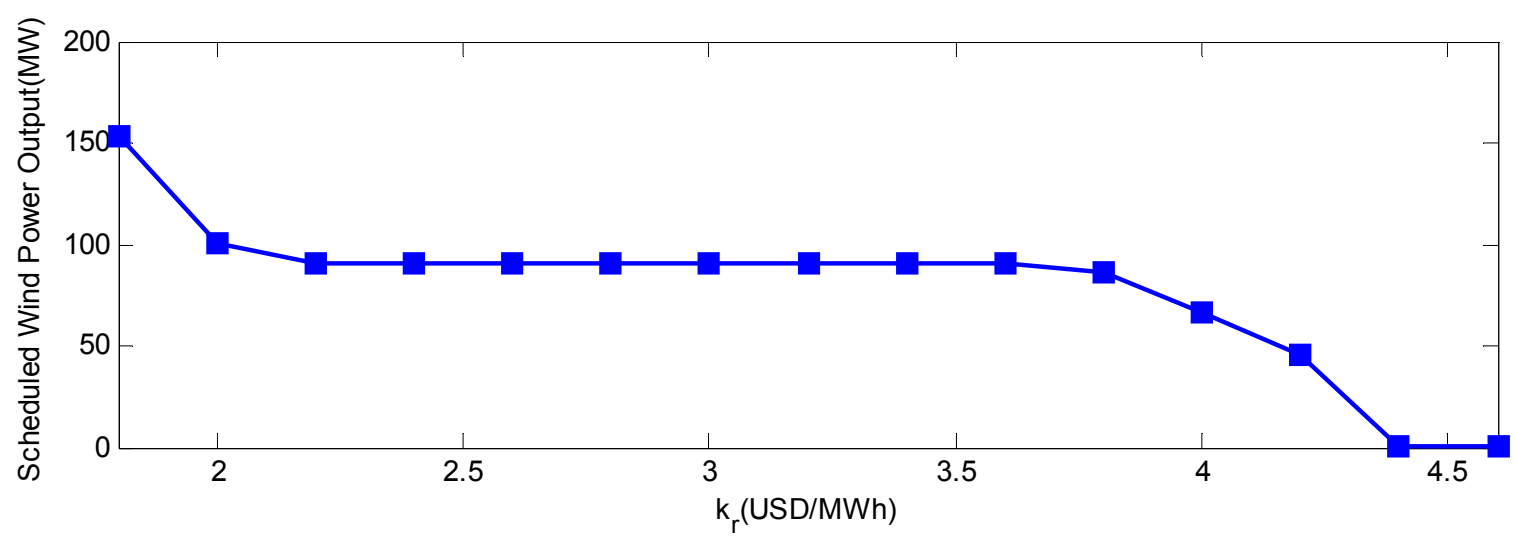

Figure 7. The required spinning reserve as a function of up reserve cost coefficient.

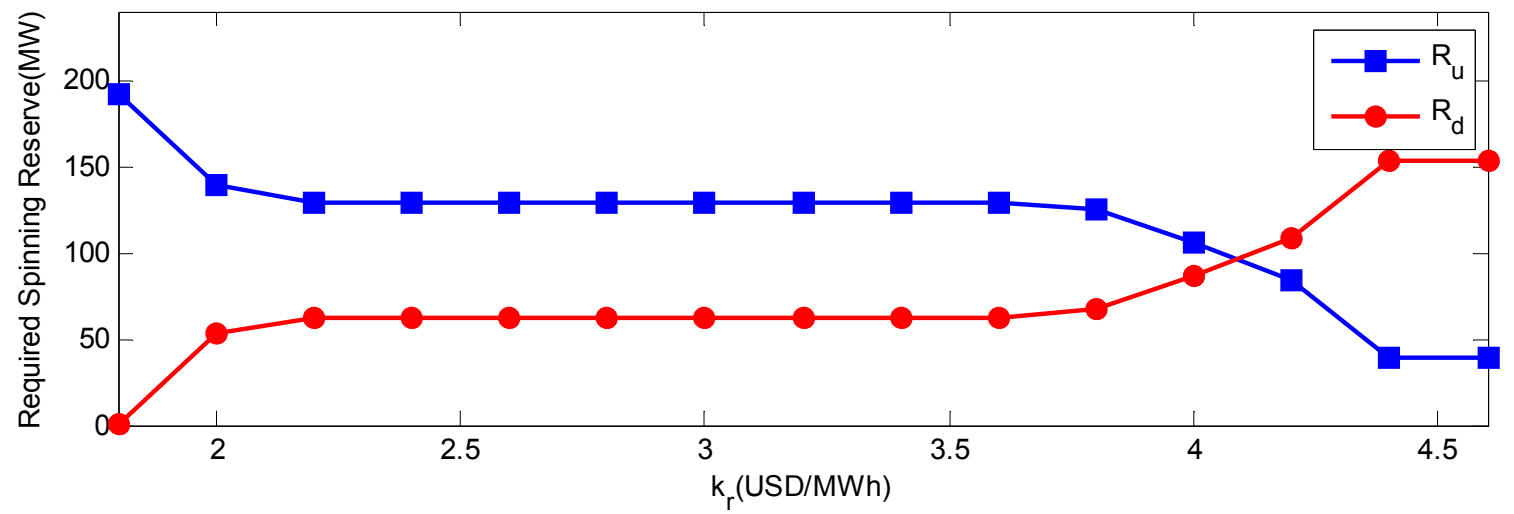

\subsection{Effects of Up Reserve Cost Coefficient on the Optimal Outputs}

Further study is to illustrate the influence of various up reserve cost coefficients on the solutions of ED problem. As shown in Figure 5, the total generation cost gradually increases as up reserve cost coefficient rises till a value of 4.4 USD/ MWh, after that it almost maintain a constant. This can be interpreted by Figures 6 and 7. Figure 6 indicates that the amount of scheduled wind power generation must be reduced to confront the rising up reserve cost coefficient. If the up reserve cost coefficient is high enough, the scheduled output will be equivalent to zero. On the contrary, the output will not increase further even when the up reserve cost coefficient is reduced to zero. Looking at Figure 7, the variation trend of up required spinning reserve by power system is almost consistent with that of scheduled wind power generation, while the reverse variation trend of down required spinning reserve can be obtained with the increasing of up reserve cost coefficient. From Figure 6 and Figure 7, it can be found that the scheduled wind power output and up/down required spinning reserve would keep constant when up reserve cost coefficient is high enough. Accordingly, the total generation cost also can’t increase any more.

\subsection{Effects of Down Reserve Cost Coefficient on the Optimal Outputs}

In this paragraph, the influence of down spinning reserve cost coefficient on the optimal solutions will be investigated. The proposed ED function is run for different values of $k_{p}$ varying from 27 USD/ 
MWh to 32 USD/MWh. When down spinning reserve cost coefficient is smaller than $30.5 \mathrm{USD} / \mathrm{MWh}$, the corresponding total generation cost and wind power output depicted in Figure 8 and Figure 9 increase respectively with the rise of down spinning reserve cost coefficient. Figure 10 demonstrates the impacts of various $k_{p}$ on the required up and down spinning reserves. The variation trend of the required up spinning reserve in this figure is similar to that of Figure 9. However, the required down spinning reserve will be decreased due to the increasing penalty cost coefficient. If the down spinning reserve cost coefficient continues to rise, the required down spinning reserve will reach zero. That means the term of down spinning reserve cost in the objective function won't have an effect on the solution of ED issue. Therefore, the total generation cost, scheduled wind power output and required up spinning reserve will also remain unchangeable.

Figure 8. The total generation cost as a function of down reserve cost coefficient.

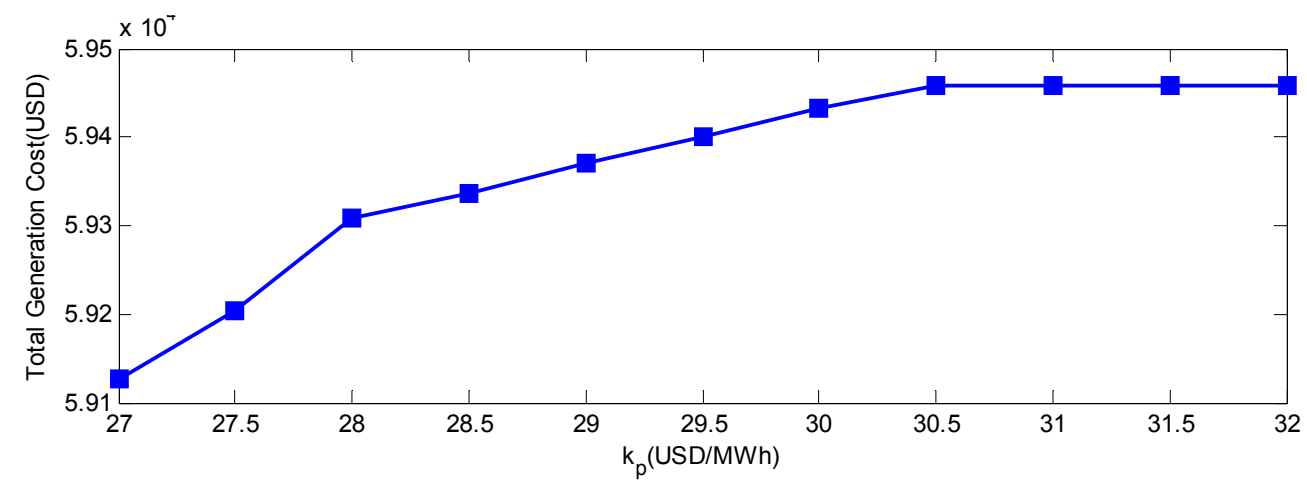

Figure 9. The scheduled wind power output as a function of down reserve cost coefficient.

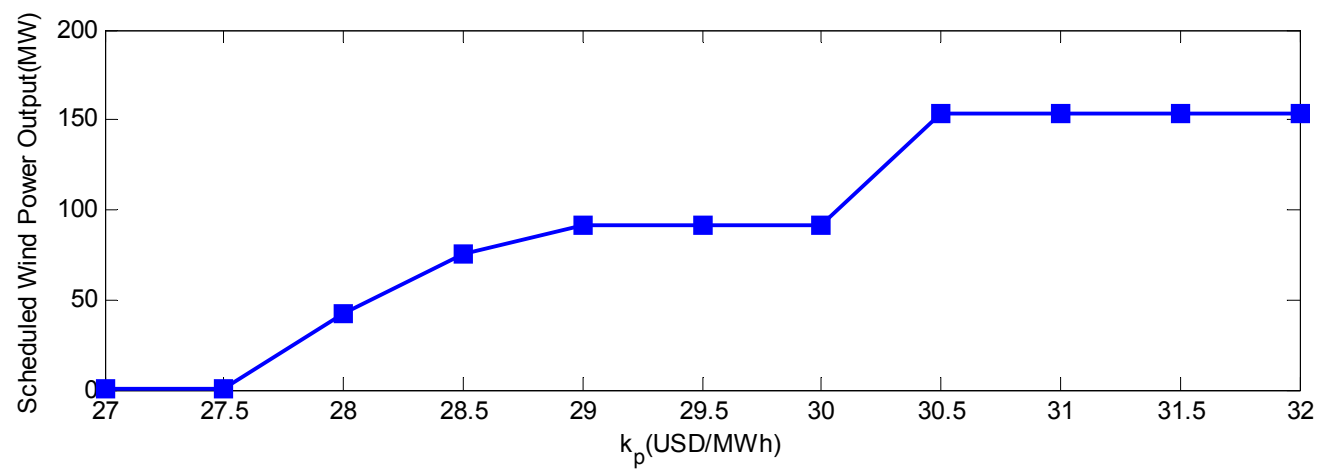

Figure 10. The required up/down spinning reserve as a function of down reserve cost coefficient.

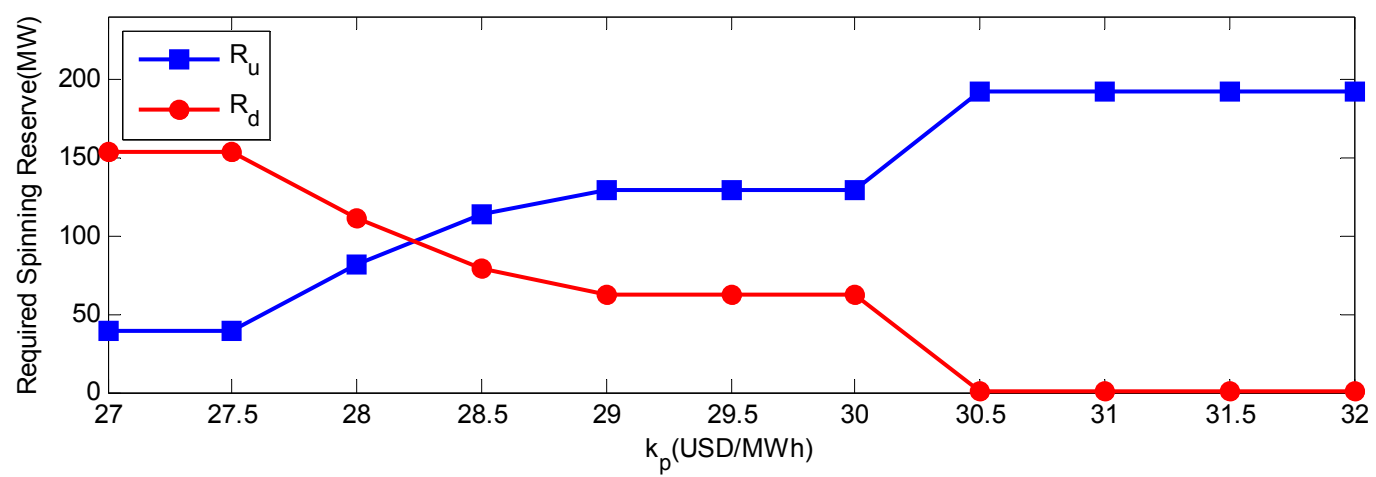




\section{Conclusions}

This paper presents a modified ED model with large-scale wind power penetration. Since stochastic wind power and other uncertain factors in system operation lead to energy deficiency or surplus, both the up and down spinning reserve are introduced in the optimization model to confront the problems of overestimation and underestimation of power generation. In the objective function, the required up and down spinning reserve costs are included, which stand for the costs caused by the risk of shedding load and penalty of wasting energy, respectively. At the same time, the corresponding risk reserve constraints are developed in order to guarantee that all the available wind power generation will be utilized within a given risk threshold. Optimal allocation of ED issue involving ten conventional generators and one wind farm power system is determined by the predictor-corrector IP method. Simulation results demonstrate that the algorithm is efficient and the solution is reasonable. The following conclusions can also be reached: (1) rising the risk threshold brings economic benefits by reducing the total generation cost, but it results in power system security being sacrificed, so system operators can choose an appropriate risk threshold to achieve the compromise between economic and security requirements. (2) The costs of required up and down spinning reserves vary with the scheduled wind power output. (3) Up and down reserve cost coefficients reflect the penalty degree of risk and waste, which are crucial parameters as risk threshold in quantifying the level of wind power scheduled.

\section{References}

1. Ummels, B.; Gibescu, C.M.; Pelgrum, E.; Kling, W.L.; Brand, A.J. Impacts of wind power on thermal generation unit commitment and dispatch. IEEE Trans. Energy Convers. 2007, 22, 44-51.

2. Lee, T.Y. Optimal spinning reserve for a wind-thermal power system using EIPSO. IEEE Trans. Power Syst. 2007, 22, 1612-1621.

3. Chen, C.L. Optimal wind-thermal generating unit commitment. IEEE Trans. Energy Convers. 2008, 23, 273-280.

4. Chen, C.L.; Lee, T.Y.; Jan, R.M. Optimal wind-thermal coordination dispatch in isolated power systems with large integration of wind capacity. Energy Conv. Manage. 2006, 47, 18-19.

5. Miranda, V.; Hang, P.S. Economic dispatch model with fuzzy wind constraints and attitudes of dispatchers. IEEE Trans. Power Syst. 2005, 20, 2143-2145.

6. Wang, L.; Singh, F.C. Tradeoff between risk and cost in economic dispatch including wind power penetration using particle swarm optimization. In Proceedings of the 2006 International Conference on Power System Technology, Chongqing, China, 5 September 2006.

7. Hong, Y.Y.; Li, C.T. Short-term real-power scheduling considering fuzzy factors in an autonomous system using genetic algorithms. Inst. Eng. Technol. 2006, 153, 684-692.

8. Hetzer, J.; Yu, D.C. An economic dispatch model incorporating wind power. IEEE Trans. Energy Convers. 2008, 23, 603-611.

9. Justus, C.G.W.; Hargraves, R.; Mikhail, A.; Graber, D. Methods for estimating wind speed frequency distributions. J. Appl. Meteorol. 1978, 17, 350-353.

10. Jabr, R.A.; Pal, B.C. Intermittent wind generation in optimal power flow dispatching. IET Gener. Transm. Distrib. 2009, 3, 66-74. 
11. Wang, J.H.; Shahidehpour, M.Z.; Li, Y. Security-constrained unit commitment with volatile wind power generation. IEEE Trans. Power Syst. 2008, 23, 1319-1327.

12. Bouffard, F.; Galiana, F.D. Stochastic security for operations planning with significant wind power generation. IEEE Trans. Power Syst. 2008, 23, 306-316.

13. Soder, L. Reserve margin planning in a wind-hydro-thermal power system. IEEE Trans. Power Syst. 1993, 8, 564-571.

14. Siahkali, H.; Vakilian, M. Stochastic unit commitment of wind farms integrated in power system. Electr. Power Syst. Res. 2010, 80, 1006-1017.

15. Doherty, R.; Malley, M.O. A new approach to quantify reserve demand in systems with significant installed wind capacity. IEEE Trans. Power Syst. 2005, 20, 587-595.

16. Lange, M. On the uncertainty of wind powr predictions-analysis of the forecast accuracy and statistical distribution of errors. Solar Energy Eng. 2005, 127, 177-184.

17. Fabbri, A.; Roman, T.G.S.; Abbad, J.R.; Quezada, V.H.M. Assessment of the cost associated with wind generation prediction errors in a liberalized electricity market. IEEE Trans. Power Syst. 2005, 20, 1440-1446.

18. Matevosyan, J.; Soder, L. Minimization of imbalance cost trading wind power on the short-term power market. IEEE Trans. Power Syst. 2006, 21, 1396-1404.

19. Zhao, M.; Chen, Z.; Blaabjerg, F. Probabilistic capacity of a grid connected wind farm based on optimization method. Renewable Energy 2006, 31, 2171-2187.

20. Valenzuela, J.; Mazumdar, M.; Kapoor, A. Influence of temperature and load forecast uncertainty on estimation of power generation production costs. IEEE Trans. Power Syst. 2000, 15, 668-674.

21. Billinton, R.; Huang, D. Effects of load forecast uncertainty on bulk electric system reliability evaluation. IEEE Trans. Power Syst. 2008, 23, 418-425.

22. Wu, Y.C.; Debs, A.; Marsten, R.E. A direct nonlinear predictor-Corrector primal-dual interior point algorithm for optimal power flows. IEEE Trans. Power Syst. 1994, 9, 876-883.

23. Zhou, W.; Peng, Y.; Sun, H. Probabilistic wind power penetration of power system using nonlinear predictor-corrector primal-dual interior-point method. In Proceedings of the 2008 IEEE Electric Utility Deregulation and Restructuring and Power Technologies Conference, Nanjing, China, 6-9 April 2008.

24. Attaviriyanupap, P.; Kita, H.; Tanaka, E.; Hasegawa, J. A hybrid EP and SQP for dynamic economic dispatch with nonsmooth fuel cost function. IEEE Trans. Power Syst. 2002, 17, 411-416.

(C) 2010 by the authors; licensee MDPI, Basel, Switzerland. This article is an open access article distributed under the terms and conditions of the Creative Commons Attribution license (http://creativecommons.org/licenses/by/3.0/). 